MATHEMATICS OF COMPUTATION

Volume 78, Number 265, January 2009, Pages 405-420

S $0025-5718(08) 02137-6$

Article electronically published on May 14, 2008

\title{
TILES IN QUASICRYSTALS WITH QUARTIC IRRATIONALITY
}

\author{
KEVIN G. HARE
}

\begin{abstract}
In 2003, Pelantová and Twarock did research into the number of, and types of, tiles found in 1-dimensional cut and project quasicrystals associated with 7-order symmetry. In this paper we extend this to symmetries of order 9 (degree 3), as well as orders 15, 16, 20 and 24 (degree 4). Some discussion of the next case, order 11 (degree 5), is given.
\end{abstract}

\section{INTRODUCTION AND DEFINITIONS}

Since their discovery in 1984 [13], quasicrystals have been the focus of considerable study [1, 6, 7, 8, 9, 10, 11]. Quasicrystals are objects that exhibit long range aperiodic order, yet despite this, exhibit much of the structure and order one comes to expect from their more common cousins, crystals. Unlike crystals, which may only exhibit rotational symmetries of orders 2, 3, 4 or 6, quasicrystals, in theory, can exhibit rotational symmetries of many other orders. (In practice, only orders 5, 8, 10 and 12 have been observed.) This paper studies the number of tiles one would expect in a quasicrystal with a "simple" rotational symmetry. The number of tiles can, to some extent, be thought of as the complexity of a quasicrystal. The results in this paper go some way to show why one would not expect higher degree symmetries to be observed experimentally.

We let $\mathcal{O}_{k}=\mathbb{Z}\left[2 \cos \left(\frac{2 \pi}{k}\right)\right]$. This is the ring of integers of the maximal real subfield of the $k$ th cyclotomic field $\mathbb{Q}\left(\mathrm{e}^{2 \pi i / k}\right)$, and the degree over $\mathbb{Q}$ of this subfield is $n=\phi(k) / 2$, where $\phi$ is the Euler phi-function. Denote the ring automorphisms of $\mathcal{O}_{k}$ by $\sigma_{1}, \ldots, \sigma_{n}$ with $\sigma_{1}$ the identity.

Definition 1.1. We define the 1-dimensional cut and project quasicrystal of degree $n$, with acceptance windows $\Omega_{2}, \ldots, \Omega_{n}$, as

$$
\Sigma_{k}\left(\Omega_{2}, \ldots, \Omega_{n}\right)=\left\{\alpha \in \mathcal{O}_{k} \mid \sigma_{j}(\alpha) \in \Omega_{j}, j=2, \ldots, n\right\} .
$$

This definition (and most of the theoretical results) can be easily generalized to use any ring of integers $\mathcal{O}$, instead of $\mathcal{O}_{k}$. For notational and stylistic reasons, we will sometimes write $\Sigma=\Sigma_{k}=\Sigma\left(\Omega_{2}, \ldots, \Omega_{n}\right)=\Sigma_{k}\left(\Omega_{2}, \ldots, \Omega_{n}\right)$, if the acceptance windows or order are either implied or not relevant.

Definition 1.2. We define $T_{k}\left(\Omega_{2}, \ldots, \Omega_{n}\right)$ to be the set of tiles, where tiles are defined as the distances between neighbouring points in the quasicrystal. Formally,

Received by the editor September 12, 2007 and, in revised form, January 10, 2008.

2000 Mathematics Subject Classification. Primary 52C23.

The research of K. G. Hare was supported, in part, by NSERC of Canada. Computational support was provided for, in part, by the Canadian Foundation for Innovation and the Ontario Research Fund. 
we order $\Sigma$ as

$$
\Sigma_{k}\left(\Omega_{2}, \ldots, \Omega_{n}\right)=\left\{\cdots<y_{-1}<y_{0}<y_{1}<y_{2}<\cdots\right\}
$$

and set

$$
T_{k}\left(\Omega_{2}, \ldots, \Omega_{n}\right)=\left\{y_{i}-y_{i-1} \mid i \in \mathbb{Z}\right\} .
$$

Again, we may write $T=T_{k}=T\left(\Omega_{2}, \ldots, \Omega_{n}\right)=T_{k}\left(\Omega_{2}, \ldots, \Omega_{n}\right)$ if the acceptance windows or the order are either implied, or not relevant.

One reason that the cut and project method is used to model quasicrystals is that the tiles tend to be not too big, and at the same time, not too small. To be precise, we make the definitions:

Definition 1.3. A set $\Lambda$ is a Delone set if it is uniformly discrete, and relatively dense. That is, if there exists $0<r \leq R$ such that all balls of radius $r$ contain at most one point in $\Lambda$, and all balls of radius $R$ contain at least one point in $\Lambda$.

Definition 1.4. A set $\Lambda$ is a Meyer set if it is a Delone set and if there exists a finite set $F$ such that $\Lambda-\Lambda \subseteq \Lambda+F$.

Fact 1.1. We have that $\Sigma$ is a Meyer set, and that $T$ has a finite number of tiles.

The above fact follows from the work of [10, Section 3].

Rewritten into our notation, the main goal in [11] was to find the possible sizes of $T_{7}\left(\Omega_{2}, \Omega_{3}\right)$. This paper extends these results to other orders and higher degrees.

The first step in determining the size of $T$ is to determine if there are relationships on the acceptance windows which ensure that the tile sets will have the same size. To do this we adopt and adapt the concept of equivalence from [11.

Definition 1.5. We call two quasicrystals $\Sigma_{k}\left(\Omega_{2}^{A}, \ldots, \Omega_{n}^{A}\right)$ and $\Sigma_{k}\left(\Omega_{2}^{B}, \ldots, \Omega_{n}^{B}\right)$ equivalent if they have the same tile set modulo rescaling by a constant factor $\lambda \in \mathcal{O}_{k}$, that is, if

$$
T_{k}\left(\Omega_{2}^{A}, \ldots, \Omega_{n}^{A}\right)=\lambda T_{k}\left(\Omega_{2}^{B}, \ldots, \Omega_{n}^{B}\right) .
$$

We get the obvious generalizations of two results in [11, following the same proofs.

Lemma 1.1 (Lemma 2.2 of [1]). Let $\Omega_{2}, \ldots, \Omega_{n}$ be bounded half-closed intervals. Then $\Sigma\left(\Omega_{2}, \ldots, \Omega_{n}\right)$ and $\Sigma\left(\Omega_{2}+a_{2}, \ldots, \Omega_{n}+a_{n}\right)$ are equivalent for any $a_{i} \in \mathbb{R}$.

Using methods similar to [11, Proposition 2.3], it can be shown that the tile sets for $\Sigma\left(\left[0, \ell_{2}\right), \ldots,\left[0, \ell_{n}\right)\right)$ and $\Sigma\left(\left[0, \ell_{2}\right], \ldots,\left[0, \ell_{n}\right]\right)$ differ by at most $n-1$ tiles for any $\ell_{j} \in \mathbb{R}$. A similar argument shows that $\Sigma\left(\left(0, \ell_{2}\right), \ldots,\left(0, \ell_{n}\right)\right)$ and $\Sigma\left(\left[0, \ell_{2}\right), \ldots\right.$, $\left.\left[0, \ell_{n}\right)\right)$ have the same tile set. As such, for this paper, we only consider half-closed intervals.

Tiles such that both end points of the tile are end points under some $\sigma_{j}$ of $\Omega_{j}$ will occur with 0 density. Tilings that contain such tiles are known as singular tilings [1, 7. For the purposes of this paper, we will only be concerned with non-singular tilings; hence we will always assume that the $\Omega_{j}$ are half-closed.

In Section 2 we discuss a technique to solve a particular computational problem that is needed for Sections 3 and 4 . In Section 3 we will show how Lemmas 1.1 and 3.1, along with the computational technique from Section 2, allow us to find a relevant area $\mathcal{L}_{R A} \subset \mathbb{R}^{n-1}$ for $\mathcal{O}_{k}$. A relevant area $\mathcal{L}_{R A}$ has the property that all $\Sigma$ based on half-closed intervals are equivalent to some $\Sigma\left(\left[0, \ell_{2}\right), \ldots,\left[0, \ell_{n}\right)\right)$ with $\left(\ell_{2}, \ldots, \ell_{n}\right) \in \mathcal{L}_{R A}$. In Section 4 we discuss how to find the tile set for a 
cut and project quasicrystal with specified $\Omega_{2}, \ldots, \Omega_{n}$. In Section 5 we show how to partition the relevant area $\mathcal{L}_{R A}$ into subregions, each of which has a constant tile set. Section [6 discusses the computational aspects that would be involved in extending the results of this paper to order 11 (the next case), as well as some experimental results. In Section 7 we combine Section 5 with Sections 3 and 4 to find all tile set sizes for points in the relevant area $\mathcal{L}_{R A}$ for specific orders. These results are summarized in Table 7.1 .

\section{Computational tools}

There is a common problem that comes up in both the analysis of determining the $\mathcal{L}_{R A}$ and, later, in determining the tile set and tile set size of $\Sigma\left(\Omega_{2}, \ldots, \Omega_{n}\right)$ for specific $\Omega_{2}, \ldots, \Omega_{n}$.

This problem can be summarized as follows.

Problem 2.1. Let $B$ be a box in $\mathbb{R}^{m}$ with edges parallel to the axes. Let $S_{i}$ be a sequence of smaller boxes in $\mathbb{R}^{m}$, again with the edges parallel to the axes. Further, let each $S_{i}$ be contained within $B$ and share at least one corner with $B$. Find:

(1) $N$ minimal (if it exists) such that

$$
\bigcup_{i=1}^{N} S_{i}=B
$$

(2) all $j \leq N$ such that

$$
\bigcup_{i=1}^{j-1} S_{i}=\bigcup_{i=1}^{j} S_{i} .
$$

To put this less formally, we want to find out how many boxes $S_{i}$ are needed to cover $B$, as well as which boxes $S_{j}$ are unused (based on the previous $S_{0}, S_{1}, \ldots, S_{j-1}$ boxes).

The algorithm used to solve this problem is best demonstrated by an example.

Example 1. Let $B=[0,6] \times[0,6]$ and let the smaller boxes be

$$
\begin{array}{ll}
S_{1} & =[0,5] \times[0,3], \quad S_{2}=[3,6] \times[0,5], \quad S_{3}=[4,6] \times[0,1], \\
S_{4} & =[0,4] \times[2,6], \quad S_{5}=[2,6] \times[4,6] .
\end{array}
$$

(a) First we subtract $S_{1}=[0,5] \times[0,3]$ from $B$. We see that

$$
B \backslash S_{1}=[5,6] \times[0,6] \cup[0,6] \times[3,6] .
$$

(b) Next we subtract $S_{2}=[3,6] \times[0,5]$ from $B \backslash S_{1}$. We see that

$$
\begin{aligned}
B \backslash\left(S_{1} \cup S_{2}\right)= & ([5,6] \times[0,6] \cup[0,6] \times[3,6]) \cap([0,3] \times[0,6] \cup[0,6] \times[5,6]) \\
= & ([5,6] \times[0,6] \cap[0,3] \times[0,6]) \cup([5,6] \times[0,6] \cap[0,6] \times[5,6]) \\
& \cup([0,6] \times[3,6] \cap[0,3] \times[0,6]) \cup([0,6] \times[3,6] \cap[0,6] \times[5,6]) \\
= & \emptyset \times[0,6] \cup[5,6] \times[5,6] \cup[0,3] \times[3,6] \cup[0,6] \times[5,6] \\
= & {[5,6] \times[5,6] \cup[0,3] \times[3,6] \cup[0,6] \times[5,6] } \\
= & {[0,3] \times[3,6] \cup[0,6] \times[5,6] . }
\end{aligned}
$$


Here we are able to remove $\emptyset \times[0,6]$ from line (2.1) as it is empty. In line (2.2), we are able to remove $[5,6] \times[5,6]$ because it is contained within $[0,6] \times[5,6]$.

(c) Next we subtract $S_{3}=[4,6] \times[0,1]$. We see through a similar calculation that

$$
B \backslash\left(S_{1} \cup S_{2} \cup S_{3}\right)=[0,3] \times[3,6] \cup[0,6] \times[5,6],
$$

which tells us that $S_{3}$ is not useful.

(d) Next we subtract $S_{4}=[0,4] \times[2,6]$ to get

$$
B \backslash\left(S_{1} \cup S_{2} \cup S_{3} \cup S_{4}\right)=[4,6] \times[5,6] .
$$

(e) Lastly we subtract $S_{5}=[2,6] \times[4,6]$ to get

$$
B \backslash\left(S_{1} \cup S_{2} \cup S_{3} \cup S_{4} \cup S_{5}\right)=\emptyset .
$$

This is represented in Figure 2.1. The parts of each $S_{i}$ that are used to cover $B$ are shaded different colours. This tells us that $N=5$ and that the only non-useful $j$ value is $j=3$.

To summarize, the main points are:

- Start by representing $B \backslash S_{j}=\bigcup_{C \in \mathfrak{C}_{j}} C$ as a union of smaller boxes.

- Inductively define $\mathfrak{A}_{j}$ as $B \backslash\left(S_{1} \cup S_{2} \cup \ldots \cup S_{j}\right)=\bigcup_{A \in \mathfrak{A}_{j}} A$ where $\mathfrak{A}_{j}$ contains no empty boxes and no box that is completely contained in another box.

- We do this by noticing that

$$
\begin{aligned}
B \backslash\left(S_{1} \cup \ldots \cup S_{j+1}\right) & =\left(B \backslash\left(S_{1} \cup \ldots \cup S_{j}\right)\right) \cap\left(B \backslash S_{j+1}\right) \\
& =\left(\bigcup_{A \in \mathfrak{A}_{j}} A\right) \cap\left(\bigcup_{C \in \mathfrak{C}_{j+1}} C\right) \\
& =\bigcup_{A \in \mathfrak{A}_{j}, C \in \mathfrak{C}_{j+1}} A \cap C \\
& =\bigcup_{A \in \mathfrak{A}_{j+1}^{\prime \prime}} A,
\end{aligned}
$$

where $\mathfrak{A}_{j+1}^{\prime \prime}=\left\{A \cap C: A \in \mathfrak{A}_{j}, C \in \mathfrak{C}_{j+1}\right\}$.

- Remove any empty box $A$ from $\mathfrak{A}_{j+1}^{\prime \prime}$ to get a new set $\mathfrak{A}_{j+1}^{\prime}$.

- Remove any box $A_{1}$ from $\mathfrak{A}_{j+1}^{\prime}$ that is completely contained in some other box $A_{2}$ in $\mathfrak{A}_{j+1}^{\prime}$ to get a new set $\mathfrak{A}_{j+1}$.

- Check whether $\mathfrak{A}_{j+1}$ is the same as $\mathfrak{A}_{j}$ to see if $j+1$ is useful.

- Check whether $\mathfrak{A}_{j+1}=\emptyset$.

This technique works reasonably well for dimensions 4 or less, which is all that we are interested in for this paper. 


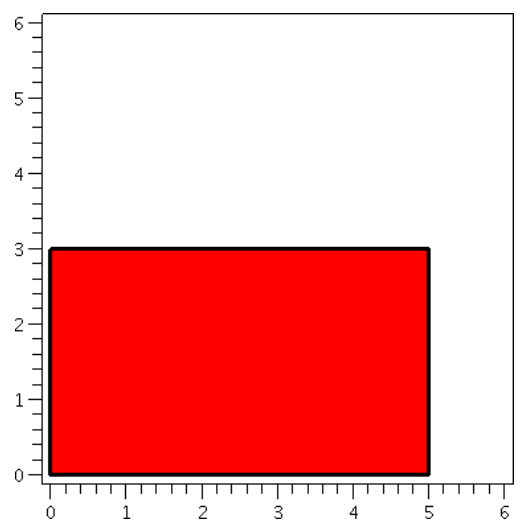

(a) $S_{1}$

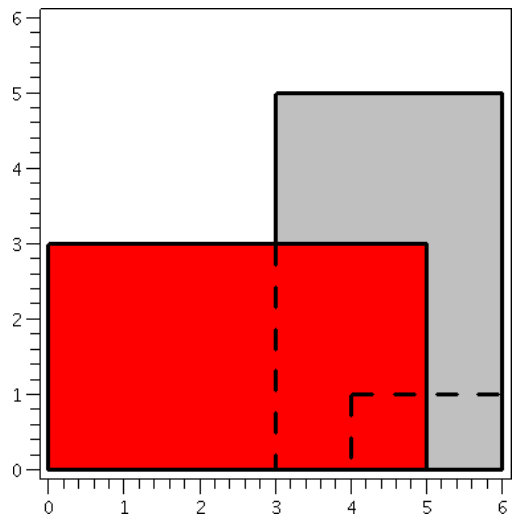

(c) $S_{1}, S_{2}$, and $S_{3}$

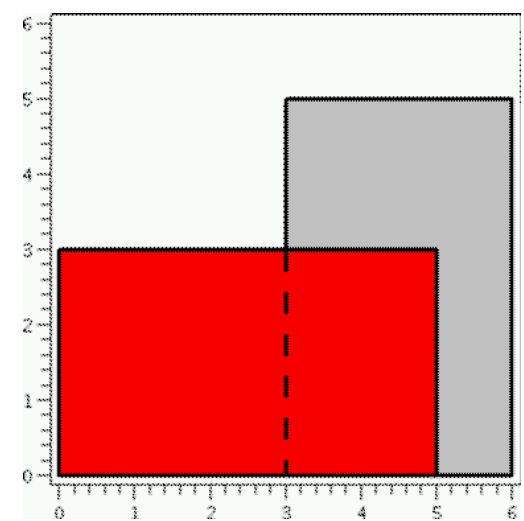

(b) $S_{1}$ and $S_{2}$

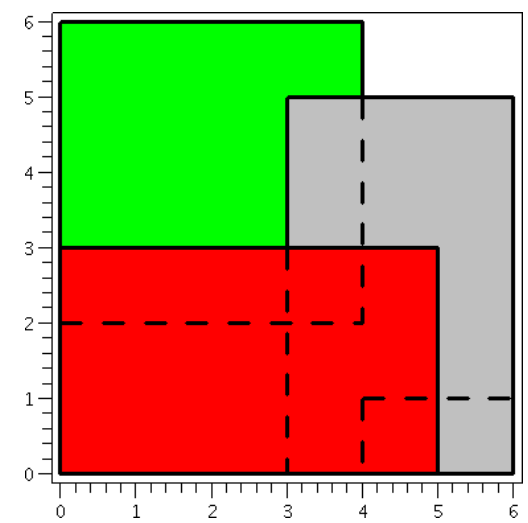

(d) $S_{1}, S_{2}, S_{3}$, and $S_{4}$

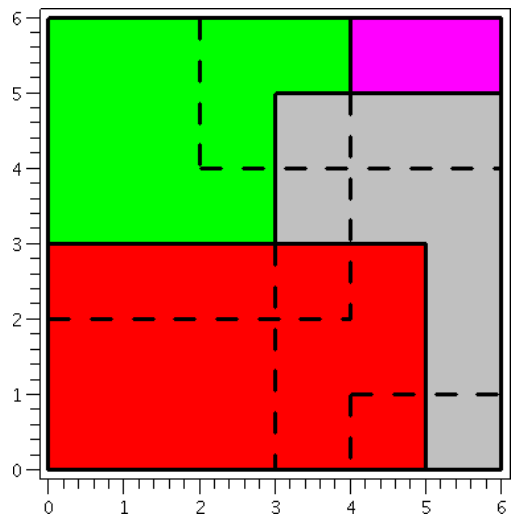

(e) $S_{1}, S_{2}, S_{3}, S_{4}$, and $S_{5}$

Figure 2.1. Covering $B$ with $S_{i}$ 


\section{Finding the Relevant area}

Based on Definition [1.5] and Lemma 1.1 we see that $T\left(\Omega_{2}, \ldots, \Omega_{n}\right)$ with halfclosed intervals is equivalent to some $T\left(\left[0, \ell_{2}\right), \ldots,\left[0, \ell_{n}\right)\right)$, with $\left(\ell_{2}, \ldots, \ell_{n}\right) \in \mathbb{R}_{+}^{n-1}$. The next result will give us a new relationship that will allow us to find a relevant area $\mathcal{L}_{R A}$ such that any $T\left(\Omega_{2}, \ldots, \Omega_{n}\right)$ with half-closed intervals is equivalent to some $T\left(\left[0, \ell_{2}\right), \ldots,\left[0, \ell_{n}\right)\right)$, with $\left(\ell_{2}, \ldots, \ell_{n}\right) \in \mathcal{L}_{R A}$, where $\mathcal{L}_{R A}$ is a closed bounded subset of $\mathbb{R}_{+}^{n-1}$.

Lemma 3.1. Let $\gamma$ be a unit in $\mathcal{O}_{k}$. That is, there exists a $\gamma^{\prime} \in \mathcal{O}_{k}$, such that $\gamma \cdot \gamma^{\prime}=1$. Then

$$
\gamma \Sigma\left(\Omega_{2}, \ldots, \Omega_{n}\right)=\Sigma\left(\sigma_{2}(\gamma) \Omega_{2}, \ldots, \sigma_{n}(\gamma) \Omega_{n}\right) .
$$

Proof.

$$
\begin{aligned}
\gamma \Sigma\left(\Omega_{2}, \ldots, \Omega_{n}\right) & =\gamma\left\{\alpha \in \mathcal{O}_{k}: \sigma_{j}(\alpha) \in \Omega_{j}\right\} \\
& =\left\{\gamma \alpha: \alpha \in \mathcal{O}_{k}, \sigma_{j}(\alpha) \in \Omega_{j}\right\} \\
& =\left\{\gamma \gamma^{\prime} \alpha: \alpha \gamma^{\prime} \in \mathcal{O}_{k}, \sigma_{j}\left(\alpha \gamma^{\prime}\right) \in \Omega_{j}\right\} \\
& =\left\{\alpha: \alpha \gamma^{\prime} \in \mathcal{O}_{k}, \sigma_{j}(\alpha) \in \sigma_{j}(\gamma) \Omega_{j}\right\} \\
& =\left\{\alpha: \alpha \in \mathcal{O}_{k}, \sigma_{j}(\alpha) \in \sigma_{j}(\gamma) \Omega_{j}\right\} \\
& =\Sigma\left(\sigma_{2}(\gamma) \Omega_{2}, \ldots, \sigma_{n}(\gamma) \Omega_{n}\right) .
\end{aligned}
$$

A value $\gamma \in \mathcal{O}_{k}$ such that there exists a $\gamma^{\prime} \in \mathcal{O}_{k}$ with $\gamma \cdot \gamma^{\prime}=1$ is called a unit of $\mathcal{O}_{k}$. The set of all units in $\mathcal{O}_{k}$ forms what is known as the unit group of $\mathcal{O}_{k}[5]$. This unit group is a finitely generated abelian group under multiplication; hence there

\begin{tabular}{|c|c|c|}
\hline Order & Root & Fundamental Units \\
\hline $\begin{array}{l}7 \\
9\end{array}$ & $\begin{array}{l}\beta=2 \cos \left(\frac{2 \pi}{7}\right) \approx 1.247 \\
\beta=2 \cos \left(\frac{2 \pi}{9}\right) \approx 1.532\end{array}$ & $\begin{array}{l}\frac{\text { Degree } 3}{\beta^{2}-1,} \beta \\
\beta-1, \quad \beta\end{array}$ \\
\hline $\begin{array}{l}15 \\
16 \\
20 \\
24\end{array}$ & $\begin{array}{l}\beta=2 \cos \left(\frac{2 \pi}{15}\right) \approx 1.827 \\
\beta=2 \cos \left(\frac{2 \pi}{16}\right) \approx 1.848 \\
\beta=2 \cos \left(\frac{2 \pi}{20}\right) \approx 1.902 \\
\beta=2 \cos \left(\frac{2 \pi}{24}\right) \approx 1.931\end{array}$ & $\begin{array}{l}\frac{\text { Degree } 4}{\beta^{3}-3 \beta,} \quad \beta-1, \quad \beta^{2}-3 \\
\beta^{2}-1, \quad \beta-1, \quad \beta^{2}+\beta-1 \\
\beta^{2}-2, \quad \beta^{2}-\beta-2, \quad \beta^{3}-\beta^{2}-3 \beta+3 \\
\beta, \quad \beta^{3}-3 \beta-1, \quad \beta^{3}-\beta^{2}-3 \beta+2\end{array}$ \\
\hline 11 & $\beta=2 \cos \left(\frac{2 \pi}{11}\right) \approx 1.682$ & $\frac{\text { Degree } 5}{\beta^{4}-3 \beta^{2}}+1, \quad \beta, \quad \beta^{2}+\beta-1, \quad \beta^{3}-2 \beta$ \\
\hline
\end{tabular}
exists a set of generators $\gamma_{1}, \gamma_{2}, \ldots, \gamma_{m}$. In this case $m=n-1$ because $\mathcal{O}_{k}$ is totally real. So all units in $\mathcal{O}_{k}$ can be written as a product $\gamma_{1}^{a_{1}} \ldots \gamma_{n-1}^{a_{n-1}}$ with $a_{j} \in \mathbb{Z}$ and $\gamma_{j}$ generators of the unit group. These generators can be found with the software package Pari, 3, and are summarized in Table 3.1. To represent the generators symbolically, we write each generator as a sum $\sum b_{i} \beta^{i}$ where $\beta=2 \cos \left(\frac{2 \pi}{k}\right)$.

TABLE 3.1. Fundamental Units 
By Lemma 3.1 we see that for any element of this unit group, say $\gamma$, we have that $\Sigma\left(\Omega_{2}, \ldots, \Omega_{n}\right)$ and $\Sigma\left(\sigma_{2}(\gamma) \Omega_{2}, \ldots, \sigma_{n}(\gamma) \Omega_{n}\right)$ are equivalent. To take advantage of lattice algorithms, and the algorithm of Section 2 , we move to logarithms. Define:

$$
w_{j}=\left(\log \left(\left|\sigma_{2}\left(\gamma_{j}\right)\right|\right), \log \left(\left|\sigma_{3}\left(\gamma_{j}\right)\right|\right), \ldots, \log \left(\left|\sigma_{n}\left(\gamma_{j}\right)\right|\right)\right)
$$

for $j$ from 1 to $n-1$.

It should be noted that there are many different $\mathcal{L}_{R A}$ and it will never be unique. We have the following lemma, which follows directly from Lemma 3.1, which will help to determine an $\mathcal{L}_{R A}$.

Lemma 3.2. A set $\mathcal{L}$ is a relevant area if $\left\{\log (\mathcal{L})+\sum w_{i} a_{i}\right\}$ with $a_{i} \in \mathbb{Z}$ tiles $\mathbb{R}^{n-1}$. I.e., the union of the $\log (\mathcal{L})+\sum w_{i} a_{i}$ covers $\mathbb{R}^{n-1}$, as the $a_{i}$ range over $\mathbb{Z}^{n-1}$, and their interiors are pairwise disjoint.

So at this point we are attempting to find any $\mathcal{L}_{R A}$ with the properties of Lemma 3.2. An easy $\log \left(\mathcal{L}_{R A}\right)$ to find is the parallelepiped given by the vectors $w_{1}, w_{2}, \ldots, w_{n-1}$. (See Figure 3.1 (a) for this parallelepiped and its translates.)

In Section 5 we will show how to partition an $\mathcal{L}_{R A}$ into subregions such that for any $\left(\ell_{2}, \ldots, \ell_{n}\right)$ in a subregion, the tile set (and hence tile set size) based on the acceptance windows $\Omega_{j}=\left[0, \ell_{j}\right)$ is constant. These subregions are boxes with edges parallel to the axes. Unfortunately, the parallelepiped found for $\log \left(\mathcal{L}_{R A}\right)$ given by the vectors $w_{1}, w_{2}, \ldots, w_{n-1}$ gives an awkward region for the $\mathcal{L}_{R A}$. (See Figure 3.1(b).) This is because we will be covering the $\mathcal{L}_{R A}$ by boxes with edges parallel to the axes, whereas this $\mathcal{L}_{R A}$ does not have edges parallel to the axes. In theory, it should be possible to use a box $B \supset \mathcal{L}_{R A}$, with edges parallel to the axes, and partition it by the methods of Section [5] Instead we will adopt the technique from [11] which will find a different $\mathcal{L}_{R A}$ such that all of its edges are parallel to the axes. The initial setup for such an object is slightly more expensive, but once it is created the partitioning of the $\mathcal{L}_{R A}$ into subregions is very easy.

We will choose our $\mathcal{L}_{R A}$ such that the upper corner is $(1,1, \ldots, 1)$. This means that after taking logarithms, that $\log \left(\mathcal{L}_{R A}\right)$ is completely in $\mathbb{R}_{\leq 0}^{n-1}$ and has a corner at $(0,0, \ldots, 0)$.

Let $\mathcal{T}$ be the natural projection from $\mathbb{R}^{n-1}$ to $\mathbb{R}^{n-2}$ by $\mathcal{T}\left(b_{2}, b_{3}, \ldots, b_{n}\right)=$ $\left(b_{3}, \ldots, b_{n}\right)$. Consider the vectors $w_{1}, w_{2}, \ldots, w_{n-1}$. Consider the fundamental region in $\mathbb{R}^{n-1}$ defined by these vectors. Call this region $F$. Clearly, $\left\{F+\sum a_{i} w_{i} \mid a_{i} \in \mathbb{Z}\right\}$ will cover $\mathbb{R}^{n-1}$. Notice that the edges of this object are not parallel to the axes. Now consider the object $\mathcal{T}(F)$. By projection, we see that $\left\{\mathcal{T}\left(F+\sum a_{i} w_{i}\right)\right.$ $\left.a_{i} \in \mathbb{Z}\right\}$ will cover $\mathbb{R}^{n-2}$. We can now take a translate of $\mathcal{T}(F)$, say by $v \in \mathbb{R}^{n-1}$, such that $\mathcal{T}(F+v)$ is in $\mathbb{R}_{<0}^{n-2}$. Call this new object $\mathcal{T}\left(F^{\prime}\right)=\mathcal{T}(F+v)$. Again, we see that $\left\{\mathcal{T}\left(F^{\prime}+\sum a_{i} w_{i}\right) \mid a_{i} \in \mathbb{Z}\right\}$ will cover $\mathbb{R}^{n-2}$. We now take $\mathcal{T}(L)$ such that $\mathcal{T}(L)$ contains $\mathcal{T}\left(F^{\prime}\right)$. Now let $L$ be the smallest $(n-2)$-dimensional box containing $\mathcal{T}\left(F^{\prime}\right)$ in the hyperplane $x_{2}=0$. Then $\left\{\mathcal{T}\left(L+\sum a_{i} w_{i}\right) \mid a_{i} \in \mathbb{Z}\right\}$ covers $\mathbb{R}^{n-2}$. Further, we see that the translates of $L$ do not overlap, as there is no lattice vector in the plane $x_{2}=0$, except $\mathbf{0}$. (A lattice vector with one component 0 corresponds to a unit $u$ with one conjugate equal to \pm 1 . In this case, $u= \pm 1$ and the lattice vector is $\mathbf{0 .}$ ) 


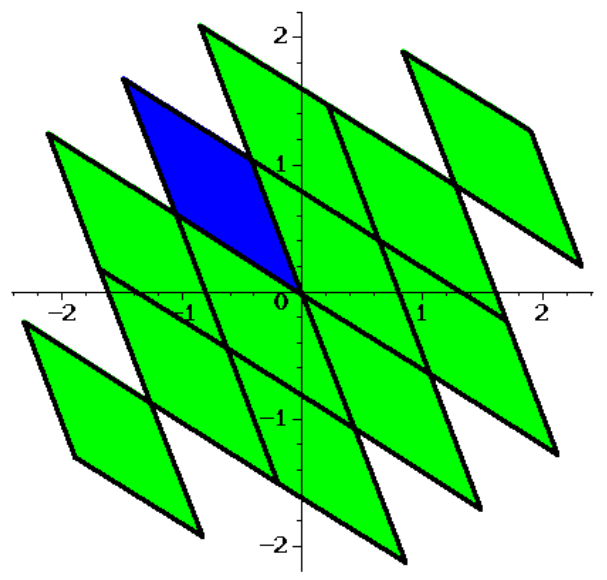

(a) Parellelepiped shaped $\mathcal{L}_{R A}$

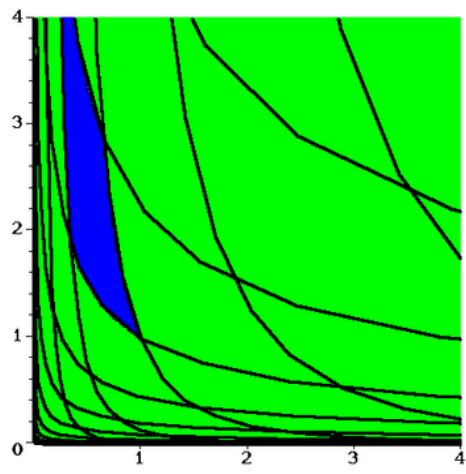

(b) Removing Logarithms

Figure 3.1. $\mathcal{L}_{R A}$ based on Parallelepiped

Given this $L$, then for all $\left(0, x_{3}, \ldots, x_{n}\right) \in L$, find the minimal $|z|$, with $z<0$, such that $\left(z, x_{3}, \ldots, x_{n}\right)$ overlaps a translate of $L$. (This is doable by the algorithm of Section [2) The $\mathcal{L}_{R A}$ is the area between $\left(0, x_{3}, \ldots, x_{n}\right)$ and $\left(z, x_{3}, \ldots, x_{n}\right)$ as $\left(0, x_{3}, \ldots, x_{n}\right)$ ranges over values in $L$. To see that we need only a finite number of different $z$ values, we note that $|z|$ is bounded by the length of $F$ under the projection $\left(x_{2}, \ldots, x_{n}\right) \rightarrow x_{2}$.

This new object, when tiled, will cover $\mathbb{R}^{n-1}$.

We will demonstrate this with an example.

Example 2. Consider the case of $\beta=2 \cos \left(\frac{2 \pi}{9}\right) \approx 1.532$ of order 9 .

This has fundamental units $\beta-1$ and $\beta$. This gives $w_{1}=(-0.427,1.058)$ and $w_{2}=(-1.058,0.631)$. As a first attempt, we can use the $\log \left(\mathcal{L}_{R A}\right)$ given by the parallelogram with vertices $(0,0), w_{1}, w_{1}+w_{2}$ and $w_{2}$. Unfortunately, when we remove the logs, the resulting $\mathcal{L}_{R A}$ is very awkward to use, from a computational point of view. (See Figures 3.1(a) and 3.1(b) for this region, and its translates in $\mathbb{R}^{n-1}$.)

Instead, we follow the outline as given above, to find an $\mathcal{L}_{R A}$ with edges parallel to the axes.

- We start with the fundamental region $F$; see Figure 3.2(a).

- We then project this region $F$ to the $y$-axis to get $\mathcal{T}(F)$; see Figure 3.2(b).

- We then translate this projection to get $\mathcal{T}\left(F^{\prime}\right)=\mathcal{T}(F+v)$, a region on the $y$-axis, and totally non-positive; see Figure 3.2 (c).

- We then use the algorithm of Section 2 to fill in the object in $\mathbb{R}^{2}$; see Figure $3.2(\mathrm{~d})$.

- The final object, when logarithms are removed, is given in Figure 3.2(e). 


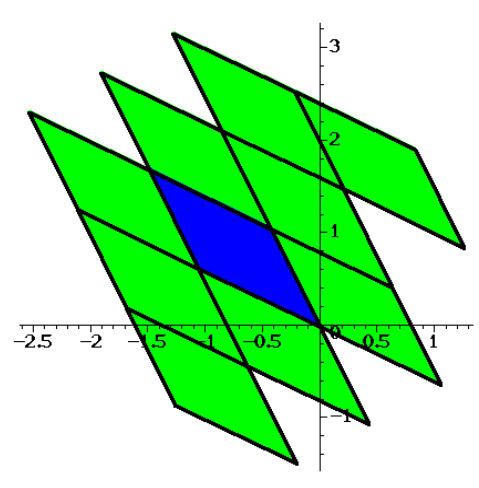

(a) Fundamental Region

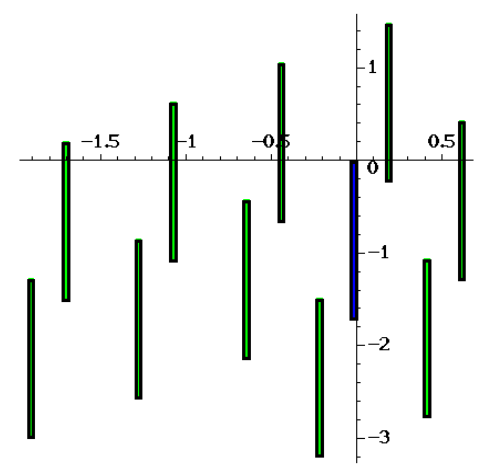

(c) Translation of Projection of Fundamental Region

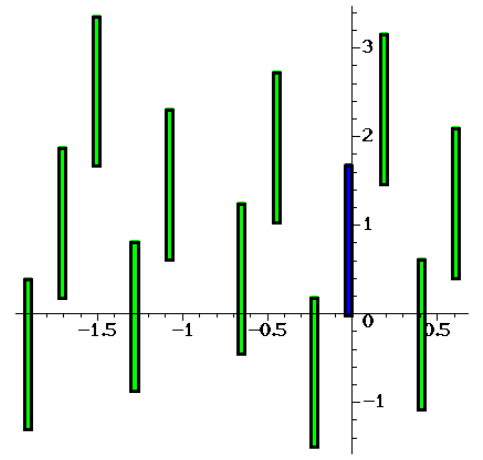

(b) Projection of Fundamental Region

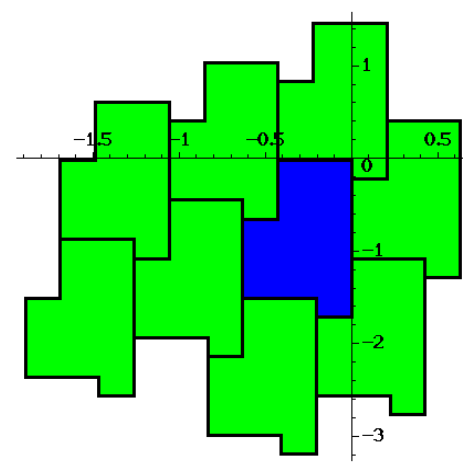

(d) Filling $\mathbb{R}^{2}$

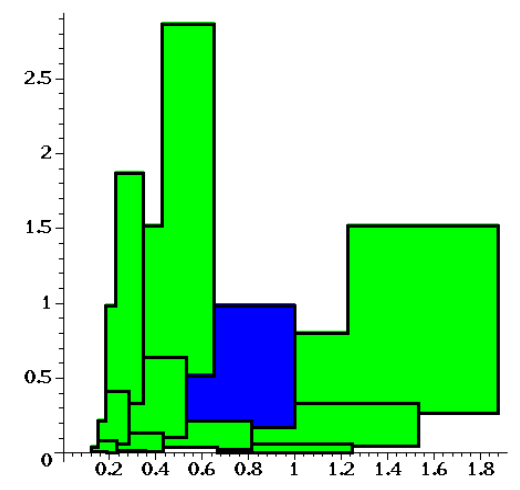

(e) Removing Logarithms

Figure 3 .2. Finding the $\mathcal{L}_{R A}$ 
TABLE 4.1. Values in $\Sigma((-1,1),(-1,1))$

\begin{tabular}{|llll|}
\hline Tile & Approximation & Box & Needed \\
\hline 0 & 0 & Not Applicable & \\
$\beta^{2}+\beta-1$ & 2.879 & $S_{1}=[0,0.347] \times[0.532,1]$ & $\mathrm{X}$ \\
$\beta^{2}+2 \beta$ & 5.412 & $S_{2}=[0.227,1] \times[0,0.185]$ & $\mathrm{X}$ \\
$2 \beta^{2}+3 \beta-2$ & 7.291 & $S_{3}=[0.574,1] \times[0.717,1]$ & $\mathrm{X}$ \\
$2 \beta^{2}+3 \beta-1$ & 8.291 & $S_{4}=[0,0.574] \times[0,0.717]$ & $\mathrm{X}$ \\
$3 \beta^{2}+5 \beta-2$ & 12.703 & $S_{5}=[0.801,1] \times[0,0.902]$ & $\mathrm{X}$ \\
$4 \beta^{2}+6 \beta-3$ & 15.582 & $S_{6}=[0.148,1] \times[0.434,1]$ & $\mathrm{X}$ \\
$4 \beta^{2}+6 \beta-2$ & 16.582 & $S_{7}=[0,0.148] \times[0,0.434]$ & \\
$5 \beta^{2}+7 \beta-4$ & 18.461 & $S_{8}=[0,0.495] \times[0.966,1]$ & \\
$5 \beta^{2}+8 \beta-3$ & 20.994 & $S_{9}=[0.375,1] \times[0,0.619]$ & $\mathrm{X}$ \\
$6 \beta^{2}+9 \beta-4$ & 23.873 & $S_{10}=[0,0.722] \times[0.151,1]$ & \\
$7 \beta^{2}+10 \beta-5$ & 26.752 & $S_{11}=[0,0.069] \times[0.683,1]$ & \\
$7 \beta^{2}+11 \beta-5$ & 28.285 & $S_{12}=[0.949,1] \times[0.335,1]$ & \\
$7 \beta^{2}+11 \beta-4$ & 29.285 & $S_{13}=[0,0.947] \times[0,0.335]$ & \\
\hline
\end{tabular}

\section{TILE SETS OF FIXED QUASICRYSTALS}

In this section we will discuss how one would find the tiles in a cut and project quasicrystal.

Consider a quasicrystal of the form $\Sigma\left(\left[0, \ell_{2}\right), \ldots,\left[0, \ell_{n}\right)\right)$. We notice that if $z \in T$, then $z=y_{2}-y_{1}$, where $y_{1}, y_{2} \in \Sigma$. This then implies that $z \in \Sigma\left(\left(-\ell_{2}, \ell_{2}\right), \ldots\right.$, $\left.\left(-\ell_{n}, \ell_{n}\right)\right)$. It is a standard integer programming problem to find all $z \in \Sigma\left(\left[-\ell_{2}, \ell_{2}\right]\right.$, $\left.\ldots,\left[-\ell_{n}, \ell_{n}\right]\right)$ with $0 \leq z \leq M$ for any finite $M$. See for instance [4, 12]. The values of $z$ corresponding to the boundary points are easy to detect and remove.

At this point, we need to see for which $z$ this is a valid tile. We do this by looking at the conjugates and the associated acceptance windows.

Example 3. Let us find the tiles for $\Sigma_{9}([0,1),[0,1))$. For convenience, let $\beta=$ $2 \cos \left(\frac{2 \pi}{9}\right) \approx 1.532$.

A quick calculation, summarized in Table 4.1, gives the values in $\Sigma_{9}((-1,1)$, $(-1,1))$ that are less than 30 . It is worth observing here that $1 \in \Sigma_{9}([-1,1],[-1,1])$ but not in $\Sigma((-1,1),(-1,1))$. As we are concerned with open intervals, not closed ones, we do not include 1 in Table 4.1

Consider the first potential tile of size $\beta^{2}+\beta-1$. If $y_{2}-y_{1}=\beta^{2}+\beta-1$, then we know that $\sigma_{2}\left(y_{2}-y_{1}\right)=\sigma_{2}\left(\beta^{2}+\beta-1\right)=0.653$. We know that both $\sigma_{2}\left(y_{2}\right)$ and $\sigma_{2}\left(y_{1}\right)$ are in $[0,1)$. So this gives a range for where $\sigma_{2}\left(y_{1}\right)$ must be. If $\sigma_{2}\left(y_{1}\right)>$ $1-0.653=0.347$, then we see that it is not possible for $\sigma_{2}\left(y_{2}-y_{1}\right)=0.653$. So we see that $\sigma_{2}\left(y_{1}\right) \in[0,0.347]$. A similar calculation shows that $\sigma_{3}\left(y_{1}\right) \in[0.532,1]$. So we need $\left(\sigma_{2}\left(y_{1}\right), \sigma_{3}\left(y_{1}\right)\right)$ to be in the box $S_{1}=[0,0.347] \times[0.5329,1]$. We say that $S_{1}$ is the box associated to the tile $\beta^{2}+\beta-1$.

For each potential tile $z \in \Sigma((-1,1),(-1,1))$ in question, and each $y_{1} \in$ $\Sigma([0,1),[0,1))$, we can find a $y_{2} \in \Sigma([0,1),[0,1))$ such that $y_{2}-y_{1}=z$, if and only if $\left(\sigma_{2}\left(y_{1}\right), \sigma_{3}\left(y_{1}\right)\right)$ is contained in a box based on $\sigma_{2}(z)$ and $\sigma_{3}(z)$.

Now this difference is not always a tile. In particular, we see that $y_{2}-y_{1}$ is not a valid tile if there always exists a $y_{3}$ with $y_{2}>y_{3}>y_{1}$. This is equivalent to saying 


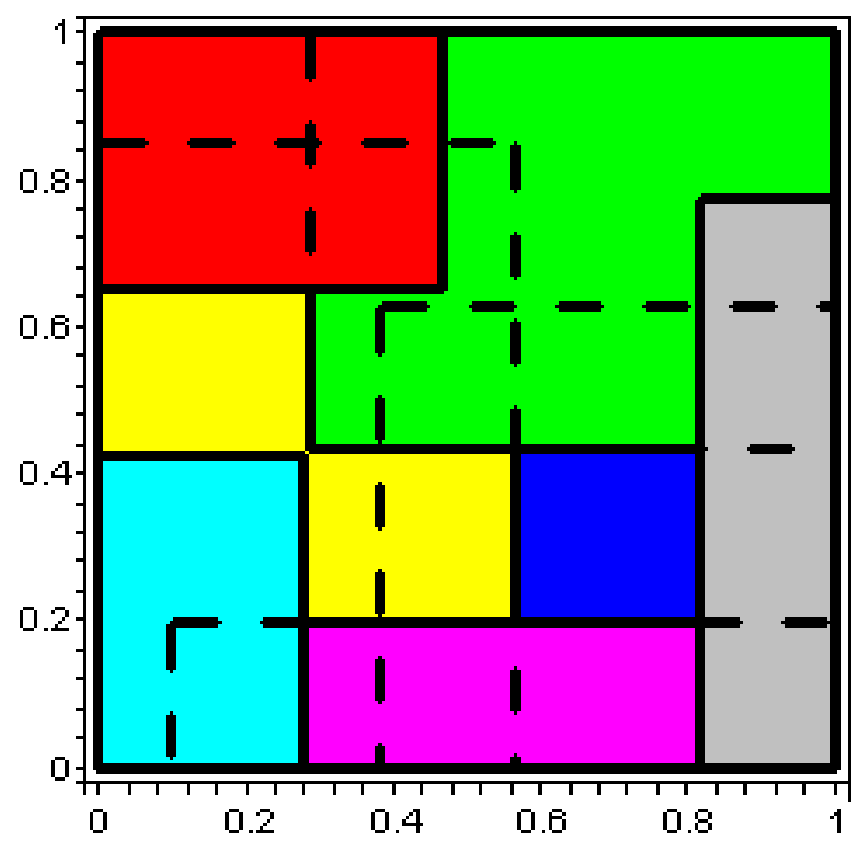

FiguRE 4.1. Tiling of order $9, \Omega_{2}=\Omega_{3}=[0,1)$

that for all $\left(\sigma_{2}\left(y_{1}\right), \sigma_{3}\left(y_{1}\right)\right)$ in the box associated to the tile $y_{2}-y_{1}$, we have that $\left(\sigma_{2}\left(y_{1}\right), \sigma_{3}\left(y_{1}\right)\right)$ is in a previous box associated to an earlier tile $y_{3}-y_{1}$.

We examine the boxes in order of their tile size. What we wish to find is:

- When the boxes eventually cover $[0,1] \times[0,1]$ and,

- For which $j$ the box $S_{j}$ is contained in the union of all previous boxes (i.e., it is an unused tile).

This is just the technique from Section 2 and a simple calculation shows that we need only the boxes $S_{1}, S_{2}, S_{3}, S_{4}, S_{5}, S_{6}$ and $S_{9}$ as indicated in Table 4.1. (See Figure 4.1.)

Thus we see that the tile set size for $\Sigma([0,1),[0,1))$ is 7 .

The general case would be done similarly to the example above. To find the valid tiles in $\Sigma\left(\left[0, \ell_{2}\right), \ldots,\left[0, \ell_{n}\right)\right)$ we compute the first few values in $\Sigma\left(\left(-\ell_{2}, \ell_{2}\right), \ldots\right.$, $\left.\left(-\ell_{n}, \ell_{n}\right)\right)$ that are greater than 0 . (We compute more as necessary, until the algorithm terminates.) After this, we consider a tile, say $z \in \Sigma\left(\left(-\ell_{2}, \ell_{2}\right), \ldots,\left(-\ell_{n}, \ell_{n}\right)\right)$, and use it to create a box $S$ that will cover part of $B=\left[0, \ell_{2}\right] \times \ldots \times\left[0, \ell_{n}\right]$. We continue with this process, choosing the tiles in order of size, until such time as the box $B$ is completely covered.

\section{Partitioning the Relevant area $\mathcal{L}_{R A}$}

In Section 3 we showed how to compute the relevant area $\mathcal{L}_{R A}$. In Section 4 we showed how one could compute the tile set (and hence the tile set size) for a fixed set of acceptance windows $\Omega_{j}$. In this section we show how to partition the $\mathcal{L}_{R A}$ into subregions such that in each subregion, the tile set (and hence tile set size) is constant.

To do this we need some notation. Let $S_{i}$ be the boxes in $\left[0, \ell_{2}\right] \times \ldots \times\left[0, \ell_{n}\right]$ associated with the tiles $t_{i} \in T$ in the quasicrystal $\Sigma\left(\left[0, \ell_{2}\right), \ldots,\left[0, \ell_{n}\right)\right)$. We need to 


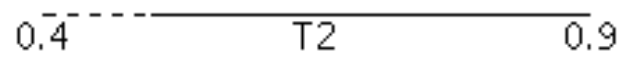

\begin{tabular}{lll}
0 & T1 & 0.4 \\
\hline
\end{tabular}

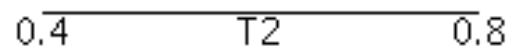

$\begin{array}{lll}0 & \mathrm{T1} & 0.3\end{array}$

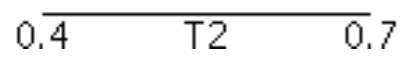

Figure 5.1. Covering $[0, \ell]$ with $[0, \ell-0.4]$ and $[0.4, \ell]$

see what happens to these boxes $S_{i}$ as the $\ell_{j}$ vary. In particular, we need to see when these regions transition from overlapping boxes that cover $B=\left[0, \ell_{2}\right] \times \ldots \times\left[0, \ell_{n}\right]$ to non-overlapping boxes that no longer cover $B=\left[0, \ell_{2}\right] \times \ldots \times\left[0, \ell_{n}\right]$.

For this, we do a very simple one-dimensional example.

Example 4. Consider $T([0,1))$ with the boxes $S_{1}=[0,0.6]$ and $S_{2}=[0.4,1]$ associated with the two tiles $t_{1}$ and $t_{2}$. These boxes cover $[0,1]$ in the sense that $[0,1]=S_{1} \cup S_{2}$. Assume further that $S_{1}$ takes precedence over $S_{2}$ in the sense that if a point is in both $S_{1}$ and $S_{2}$, then it is associated with the tile $t_{1}$ instead of $t_{2}$.

For a general tile set $T([0, \ell))$, a point $x$ is associated with $t_{1}$ if $x \in[0, \ell-0.4]$, and is associated with $t_{2}$ if $x \in[0.4, \ell]$ and is not in [0, $\left.\ell-0.4\right]$. Figure 5.1 gives examples of $S_{1}$ and $S_{2}$ as they cover (or fail to cover) [0, $\left.\ell\right]$ for $\ell=0.7,0.8,0.9$ and 1.0. We see that for $0.8 \leq \ell \leq 1.0$ the tiles $t_{1}$ and $t_{2}$ are the only tiles of importance. For $\ell<0.8$ we see that $S_{1}$ and $S_{2}$ no longer cover [0, $\left.\ell\right]$; hence we would have at least one new additional tile.

In Section 3 we found an $\mathcal{L}_{R A}$ such that every quasicrystal is equivalent to a quasicrystal using this $\mathcal{L}_{R A}$. We now wish to subdivide this $\mathcal{L}_{R A}$ into subregions such that for any $\left(b_{2}, \ldots, b_{n}\right)$ in one of these subregions we have that $\Sigma\left(\left[0, b_{2}\right), \ldots,\left[0, b_{n}\right)\right)$ has a constant tile set. To do this, we must determine, for each pair of tiles, when their associated boxes transition from overlapping to non-overlapping. So for the example above, $[0, \ell-0.4]$ and $[0.4, \ell]$ transition from overlapping to non-overlapping when $\ell=0.8$. In some cases, boxes will never overlap, so we need not consider 


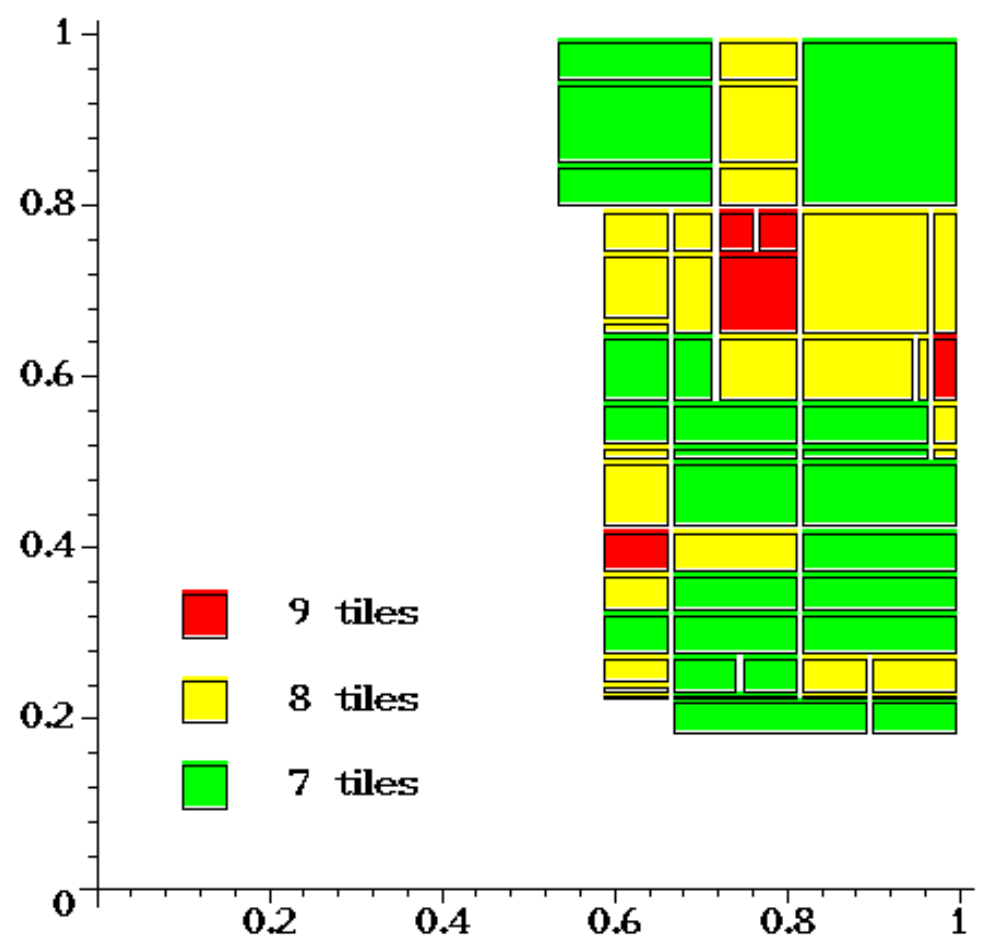

Figure 5.2. Partition for 9

these pairs. This check must be done for each direction, and each pair of overlapping boxes. We then take, for each direction, the maximal transition point. This will give us a subregion on which the tile set is constant. For the example above, this would say that for any $\ell \in[0.8,1.0]$ the tile set of $\Sigma([0, \ell))$ is the same. Beyond this transition point, we would need to recompute the tile set. We then iterate this process. To do this, consider first the acceptance windows $\Omega_{j}=\left[0, \ell_{j}\right)$ upon which we found the tile set. (For the first iteration of this algorithm, this typically has $\ell_{j}=1$.) For each direction, we find $\kappa_{j}$ such that $\kappa_{j}$ is the maximal value of a transition in this direction. This tells us that for all $\kappa_{j} \leq b_{j} \leq \ell_{j}$, the tile set on the acceptance window $\left[0, b_{2}\right),\left[0, b_{3}\right), \ldots,\left[0, b_{n}\right)$ is fixed. So this tells us that $\left[\kappa_{2}, \ell_{2}\right] \times \ldots \times\left[\kappa_{n}, \ell_{n}\right]$ is a subregion of the $\mathcal{L}_{R A}$ that has a fixed tile set. We then check the subregions

$$
\left(\left[0, \ell_{2}\right] \times \ldots \times\left[0, \ell_{i-1}\right] \times\left[0, \ell_{i}-k_{i}\right] \times\left[\ell_{i+1}-k_{i+1}, \ell_{i+1}\right] \times\left[\ell_{n}-k_{n}, \ell_{n}\right]\right) \cap \mathcal{L}_{R A}
$$

of the $\mathcal{L}_{R A}$, for $i=2,3, \ldots, n$. It should be noted that if we are checking a subregion of the $\mathcal{L}_{R A}$, say $\left[c_{2}, \ell_{2}\right] \times \ldots \times\left[c_{n}, \ell_{n}\right]$, and we find that the transition value $\kappa_{i}<c_{i}$, then this says that there is no transition value in the appropriate range that we need to worry about. Hence there is no need to recurse in this direction. We continue this process until the $\mathcal{L}_{R A}$ is fully partitioned. See, for example, Figure 5.2 


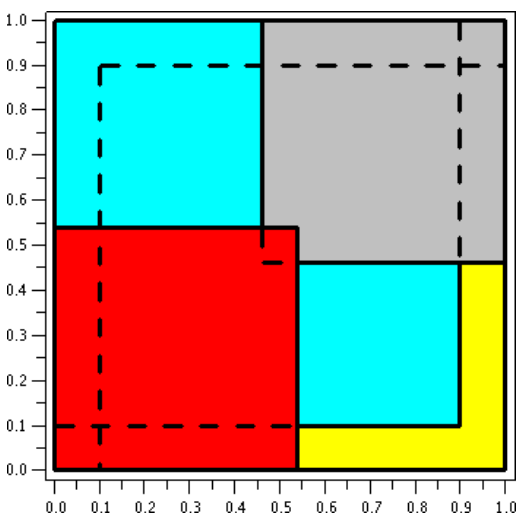

(a) $\ell=1$

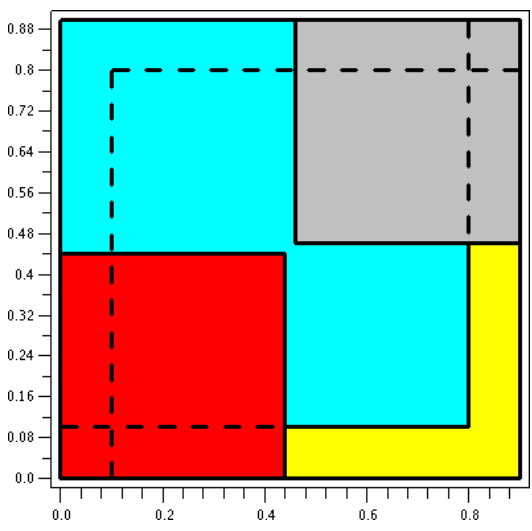

(b) $\ell=0.9$

FiguRE 5.3. Non-useful transition

It should be noted that occasionally a partition is made overly fine. This can occur when the two tiles that transition from overlapping to non-overlapping do not actually change the tile set when doing so. See, for example, Figure 5.3. It was decided not to check for these cases.

We now have all of the necessary tools to determine the tile set sizes. The results are summarized in Table 7.1 .

\section{ORDER 11}

For the previous orders $(k=7,9,15,16,20$ and 24$)$, we first determined the relevant area $\mathcal{L}_{R A}$. Next we partitioned the $\mathcal{L}_{R A}$ into subregions and computed the tile sizes for each of these subregions.

For order 11 this was deemed to be infeasible. If we compare the size of $\left[\log \left(\ell_{2}\right), \log (1)\right] \times \ldots \times\left[\log \left(\ell_{d}\right), \log (1)\right]$ with $\log \left(\mathcal{L}_{R A}\right)$, then we get a rough estimate of the number of subregions that need to be examined. Based on this, it was determined that a complete computation of order 11 would be computationally unattainable.

Based on a random sampling of 1500 subregions, chosen uniformly on $\log \left(\mathcal{L}_{R A}\right)$, we found tile set sizes for all values between 35 through 64 . This is most likely not all of them. For example, for the case when $k=20$ we have that the tile set sizes are between 15 and 30, but the tile set sizes that are within the first standard deviation are only 20 to 23 . In fact, only 10 examples where the tile set size was 30 were found (out of a possible 29,564 subregions). For this reason, it is unlikely that any random sampling of order 11 will find the extremes in the possible tile set sizes.

From a practial point of view, cut and project quasicrystals of order 11 (or higher degree orders) will probably not be recognizable in nature, as most people would not be able to observe the existence of a pattern with this large a number of tiles, and it would have the appearance of randomness. 
TABLE 6.1. Estimated number of tiles, versus actual

\begin{tabular}{|c|c|c|c|c|}
\hline Order & $\left|\log \left(B_{1}\right)\right|$ & $\log \left(\mathcal{L}_{R A}\right)$ & $\frac{\left|\log \left(\mathcal{L}_{R A}\right)\right|}{\left|\log \left(B_{1}\right)\right|}$ & Number of Subregions \\
\hline \multicolumn{5}{|c|}{ Degree 3} \\
\hline 7 & 0.010807 & 0.525454 & 48.6217 & 45 \\
\hline 9 & 0.045422 & 0.849287 & 18.6974 & 55 \\
\hline \multicolumn{5}{|c|}{ Degree 4} \\
\hline 15 & $0.88224 \mathrm{e}-5$ & 1.165455 & $132,101.2$ & 16,993 \\
\hline 16 & $0.94546 \mathrm{e}-4$ & 2.441795 & $25,826.6$ & 36,696 \\
\hline 20 & $0.24383 \mathrm{e}-4$ & 1.852811 & $75,986.5$ & 29,564 \\
\hline 24 & $0.34517 \mathrm{e}-4$ & 2.660899 & $77,088.8$ & 55,814 \\
\hline \multicolumn{5}{|c|}{ Degree 5} \\
\hline 11 & $0.47100 \mathrm{e}-7$ & 1.635694 & $34,727,742.5$ & $?$ \\
\hline
\end{tabular}

\section{Summary}

This paper formalizes, and explains how to automate, the techniques in [11. The results of all degree 3 and 4 cut and project quasicrystals are summarized in Table 7.1. These computations were done in $\mathrm{C}++$ with $20,40,80$ and then 160 digits of accuracy, under which the results remained stable. This was done using the ARPREC package of David Bailey, et al. [2]. After the $\mathcal{L}_{R A}$ was partitioned into subregions, each subregion was tested again, using a Maple implementation, to check that the number of tiles stayed the same. So, for each subregion of the $\mathcal{L}_{R A}$, the tile set was computed using both $\mathrm{C}++$ using the ARPREC package, and using code written in Maple. This was done as a means of double checking the solutions. The relative slowness of Maple was balanced in this case by the fact that the calculations were naively parallelizable and could be distributed across multiple computers.

Based upon the comments in Section 6, it is unlikely that these techniques can be useful if used for other 1-dimensional cut and project quasicrystals of higher degree based on closed or half-closed intervals. That being said, it should be possible to use these techniques to study cut and project quasicrystals of higher dimensions, or those based on multiple intervals.

TABLE 7.1. Tiles set sizes, and number of subregions

\begin{tabular}{|lll|}
\hline Order & \multicolumn{1}{l}{ Number of Subregions } & Number of non-singular Tiles \\
\hline 7 & 45 & $\frac{\text { Degree } 3}{7 \text { to } 9}$ \\
9 & 55 & 7 to 9 \\
& & Degree 4 \\
15 & 16,993 & 15 to 31 \\
16 & 36,696 & 15 to 26 \\
20 & 29,564 & 15 to 30 \\
24 & 55,814 & 15 to 28 \\
& & $\frac{\text { Degree } 5}{31}$ \\
& 35 million $(?)$ & contains 35 through 64. \\
\hline
\end{tabular}




\section{ACKNOWLEDGMENTS}

I would like to thank Jiri Patera who got me interested in this topic. I would also like to acknowledge Shuk-Ying (Janet) Yeung who wrote the initial version of the algorithm in Section 2 as part of an Undergraduate Summer Research Assistantship in the summer of 2005. Additionally I would like to thank Warren Hare, with whom I had many useful discussions concerning Section 2

I would also like to thank the anonymous (and very patient) referee, who made a number of very useful suggestions that improved the presentation of these results.

\section{REFERENCES}

[1] M. Baake. A guide to mathematical quasicrystals. ArXiv Mathematical Physics e-prints, January 1999

[2] David H. Bailey, Yozo Hida, Xiaoye S. Li, and Brandon Thompson. Arprec: An arbitrary precision computation package. Tech Technical Report LBNL-53651, Lawrence Berkeley National Laboratory, 2002. Software available from World Wide Web at (http://crd.lbl.gov/ dhbailey/mpdist). Technical Report available from World Wide Web at (http://repositories.cdlib.org/lbnl/LBNL-53651).

[3] The PARI Group. Pari/gp. http://pari.math.u-bordeaux.fr/, 2006.

[4] Laurent Habsieger and Bruno Salvy. On integer Chebyshev polynomials. Math. Comp., 66(218):763-770, 1997. MR:1401941 (97f:11053)

[5] Daniel A. Marcus. Number fields. Springer-Verlag, New York, 1977. Universitext. MR0457396 $(56: 15601)$

[6] Z. Masáková, J. Patera, and E. Pelantová. Minimal distances in quasicrystals. J. Phys. A, 31(6):1539-1552, 1998. MR1629637 (99i:82038)

[7] Z. Masáková, J. Patera, and J. Zich. Classification of Voronoi and Delone tiles in quasicrystals. I. General method. J. Phys. A, 36(7):1869-1894, 2003. MR1960699 (2004a:52041)

[8] Zuzana Masáková, Jiří Patera, and Edita Pelantová. Self-similar Delone sets and quasicrystals. J. Phys. A, 31(21):4927-4946, 1998. MR1630499 (99k:52033)

[9] Zuzana Masáková, Edita Pelantová, and Milena Svobodová. Characterization of cut-andproject sets using a binary operation. Lett. Math. Phys., 54(1):1-10, 2000. MR.1846718 (2002c:52027)

[10] R. V. Moody. Model sets: A survey. From Quasicrystals in More Complex Systems, pages 145-166, 2000. F. Axel, F. Dénoyer and J. P. Gazeau, eds.

[11] E. Pelantová and R. Twarock. Tiles in quasicrystals with cubic irrationality. J. Phys. A, 36(14):4091-4111, 2003. MR1984536 (2005b:53066)

[12] A. Schrijver. Theory of linear and integer programming. John Wiley \& Sons Ltd., Chichester, 1986. A Wiley-Interscience Publication. MR874114 (88m:90090)

[13] D. Shechtman, I. Blech, D. Gratias, and J.W. Cahn. Metallic phase with long-range orientational order and no translational symmetry. Phys. Rev. Lett., 53(20):1951-1953, 1984.

Department of Pure Mathematics, University of Waterloo, Waterloo, Ontario, CANADA, N2L 3G1

E-mail address: kghare@math. uwaterloo.ca 